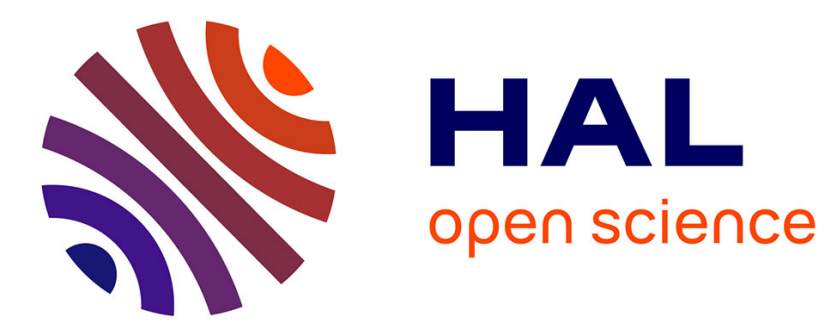

\title{
MAP Estimation of Network-Coded Correlated Sources
}

Lana Iwaza, Michel Kieffer, Khaldoun Al Agha

\section{To cite this version:}

Lana Iwaza, Michel Kieffer, Khaldoun Al Agha. MAP Estimation of Network-Coded Correlated Sources. International Conference on Advanced Technologies for Communications, Dec 2012, Hanoi, Vietnam. pp.1-4. hal-00780793

\section{HAL Id: hal-00780793 https://hal.science/hal-00780793}

Submitted on 24 Jan 2013

HAL is a multi-disciplinary open access archive for the deposit and dissemination of scientific research documents, whether they are published or not. The documents may come from teaching and research institutions in France or abroad, or from public or private research centers.
L'archive ouverte pluridisciplinaire $\mathbf{H A L}$, est destinée au dépôt et à la diffusion de documents scientifiques de niveau recherche, publiés ou non, émanant des établissements d'enseignement et de recherche français ou étrangers, des laboratoires publics ou privés. 


\title{
MAP Estimation of Network-Coded Correlated Sources
}

\author{
Lana Iwaza*†, Michel Kieffer* $^{* \dagger}$, and Khaldoun Al-Agha ${ }^{\dagger}$ \\ ${ }^{*}$ L2S- CNRS-SUPELEC-Univ Paris-Sud, 3 rue Joliot-Curie, F-91192 Gif-sur-Yvette Cedex \\ Email: lana.iwaza@1ss.supelec.fr \\ ${ }^{\dagger}$ on leave at LTCI-CNRS-TelecomParisTech, 46 rue Barrault, F-75014 Paris \\ Email: kieffer@1ss.supelec.fr \\ ${ }^{\ddagger}$ LRI-CNRS-Univ Paris-Sud, Batiment 490 - Université Paris-Sud, F-91405 Orsay Cedex \\ Email: alagha@1ri.fr
}

\begin{abstract}
This paper considers a wireless sensor network (WSN) in which sensors measure spatially correlated data and transmit these data to some data processing sink. Random Linear Network Coding (RLNC) is performed at the intermediate nodes of the network. A Maximum a Posteriori (MAP) estimator is considered at the sink to exploit the spatial correlation between data samples and provide a reconstruction of the data, even if not enough network-coded packets have been received, which usually makes network decoding very difficult. Experimental results show that with the proposed MAP estimator, the reconstruction quality increases gracefully with the number of received packets.
\end{abstract}

\section{INTRODUCTION}

The introduction of the concept of Network Coding (NC) [2] broke with the traditional assumption that intermediate network nodes can only forward incoming information streams. $\mathrm{NC}$ allows them to mix packets by performing basic operations such as linear combinations over finite fields of the content of packets [13]. In theory, NC permits to achieve the multicast capacity of a network. Significant throughput gains, in wired and wireless networks, are observed in practice [12], [5]. This is mainly due to the ability of NC to exploit wireless broadcast and to take advantage of opportunistic reception.

The upsurge of sensor networks in the recent years directed researchers towards the design and implementation of lowcomplexity sensing techniques, along with efficient solutions for information collection. The transmission of information between sensors and to a data collection sink is typically performed in a distributed manner, mainly on ad-hoc or overlay mesh network topologies. Distributed source coding (DSC) is one enabling technology for sensor networks, as it provide insights to perform distributed compression without coordination between sensors [7]. When sensors provide spatially correlated data, these data may be compressed, e.g., by channel codes [15], [18] and eventually jointly decoded at the data collection sink [16], [11].

However, this solution does not allow to fully exploit the network capacity, moreover, it requires the sensors to be aware of the correlation level between the data they produce and the data produced by neighboring sensors. In this context, DSC based on NC schemes [3], [10], [17] is known to be an efficient method for building distributed data gathering algorithms in networks with channel and source diversity. However, wireless networks are often subject to dynamic changes, and consequently to packet losses. While NC provides some robustness against packet losses, this property is linked to the reception of a sufficient number of linearly independent packets at the sink. In fact, network codes are all or nothing codes, i.e., usually, no decoding is possible unless the number of linearly independent packets at the sink is at least equal to the number of source packets being network coded.

This drawback is quite annoying, especially for delaysensitive applications, where timing constraints lead to the absence of reception of some of the packets and consequently to difficulties in decoding the transmitted sources. Compressed sensing [8], [4] and its distributed variant [14] is an alternative solution that allows to perform an approximate reconstruction of the source by exploiting some sparsity properties. However, this approach requires network codes over real fields [14], and does not apply to finite field network codes, better suited to transmission by wireless sensors of quantized data.

This paper provides an approximate estimation of spatially correlated data collected in a WSN when not enough networkcoded packets are received at the decoder side. It exploits the spatial correlation between transmitted data. The temporal correlation between successive samples collected by the sensors is not considered here. If such correlation exists, it can easily be removed via a decorrelating transform such as a DCT. A MAP estimator is provided to reconstruct the source samples based on the correlation between data contained in the networkcoded packets collected at the sink. The MAP estimator is not straightforward, since it has to cope with measurements which are linear combinations in a Galois field of the data to be estimated and for a correlation between these quantities which is expressed in the real field. The proposed coding scheme is introduced in Section II. The MAP estimator is derived in Section III, and simulation results are presented in Section IV. Some conclusions are drawn in Section V.

\section{CODING AND TRANSMISSION SCHEME}

Consider a set of $k$ wireless sensors spatially spread over some two-dimentionnal area. The location of the $i$-th sensor is 
denoted by $\boldsymbol{\theta}_{i} \in \mathbb{R}^{2}$. It measures some physical quantity $x_{i}$, assumed to be the realization of some random variable $X_{i}$. The vector $\mathbf{x}=\left(x_{1}, \ldots, x_{k}\right) \in \mathbb{R}^{k}$ gathering all measurements to be the realization of a zero-mean Gaussian vector $\mathbf{X}$ with covariance matrix

$$
\Sigma=\left[\begin{array}{ccccc}
\sigma^{2} & \sigma^{2} e^{-\lambda d_{1,2}^{2}} & \cdots & \cdots & \sigma^{2} e^{-\lambda d_{1, k}^{2}} \\
\sigma^{2} e^{-\lambda d_{1,2}^{2}} & \sigma^{2} & & & \sigma^{2} e^{-\lambda d_{2, k}^{2}} \\
\vdots & \ddots & \sigma^{2} & & \vdots \\
\vdots & & & \sigma^{2} & \sigma^{2} e^{-\lambda d_{k-1, k}^{2}} \\
\sigma^{2} e^{-\lambda d_{1, k}^{2}} & \cdots & \cdots & \cdots & \sigma^{2}
\end{array}\right]
$$

where $\sigma^{2}$ is the variance of each source, $\lambda$ is some constant, and $d_{i, j}=\sqrt{\left(\theta_{i, 1}-\theta_{j, 1}\right)^{2}+\left(\theta_{i, 2}-\theta_{j, 2}\right)^{2}}$ is the distance between Sensors $i$ and $j$. Prior to transmission, each $x_{i}$ is quantized with a $q$-level uniform scalar quantizer $Q: \mathbb{R} \rightarrow \mathbb{F}_{q}$ with stepsize $\Delta$ to get $z_{i} \in \mathbb{F}_{q}$. Each sensor then performs a linear combination of the packet containing its own quantized measurement with the packets it receives from its neighbors using RLNC. The new coded packet is transmitted to the neighboring nodes. This process is repeated until the sink considers that it has received enough packets to be able to recover the data with the desired quality.

Assume that the data processing sink has received $m \leqslant k$ linearly independent network-coded packets. It is very likely that it has received more packets, but it keeps only linearly independent informative packets. The content of the received packets is grouped in a vector $\mathbf{p} \in \mathbb{F}_{q}^{m}$. The effect of network coding is represented by the network coding matrix $A \in \mathbb{F}_{q}^{m \times k}$ linking $\mathbf{p}$ and $\mathbf{z}$ as follows

$$
\mathbf{p}=A \mathbf{z} .
$$

Each network-coded packet contains the result of the linear combinations of the quantized source samples $z_{i}$, in addition to the global coding coefficients which are stored in an extra header added to each packet [6]. Therefore, the coding matrix $A$ is known by the sink. The measurement, quantization, network coding, and estimation scheme is represented in Figure 1. Note that even if $\mathbf{x} \in \mathbb{R}^{k}$ is a vector, each of its entries is spatially spread.

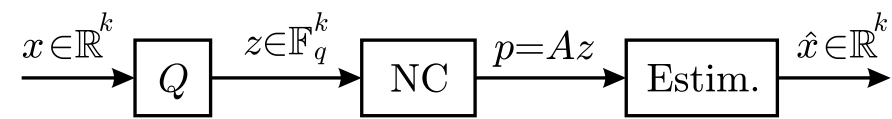

Fig. 1. Block diagram of the proposed system

The receiver has to evaluate an estimate $\widehat{\mathbf{x}}$ of $\mathbf{x}$ from the received packets $\mathbf{p}$ using the fact that the entries of $\mathbf{x}$ are correlated. For that purpose, $\Sigma$ is assumed to be known by the sink.

\section{ESTIMATION OF THE SOURCE SAMPLES}

Instead of estimating directly $\mathbf{x}$, one aims at performing first an estimate $\widehat{\mathbf{z}}$ of the vector $\mathbf{z}=\left(z_{1}, \ldots, z_{k}\right)^{T}$ of quantization indexes based on the received network-coded packets $\mathbf{p}=$ $\left(p_{1}, \ldots, p_{m}\right)^{T}$. Then, an estimate $\widehat{\mathbf{x}}$ for $\mathbf{x}$ is easily obtained from $\widehat{\mathbf{z}}$ by inverse quantization.

Provided that the scalar quantizer $Q$ is well designed, there exists two constants $\alpha$ and $\beta$ such that the reconstructed sample after inverse quantization may be expressed as $\alpha z_{i}+\beta$ and such that

$$
\begin{cases}x_{i}-\left(\alpha z_{i}+\beta\right) \leq \Delta / 2, & i=1 \ldots k \\ -x_{i}+\left(\alpha z_{i}+\beta\right) \leq \Delta / 2, & i=1 \ldots k\end{cases}
$$

Moreover, exploiting the network coding matrix, one has

$$
p_{\mu}=\sum_{j=1}^{k} a_{\mu j} z_{j}, \mu=1 \ldots m .
$$

where $a_{\mu j}$ are the global encoding coefficients of the received network-coded packets corresponding to the entries of $A$.

\section{A. Enough network-coded packets are received}

The vector $\mathbf{z}$ to estimate contains $k$ unknowns. When enough network-coded packets is received, i.e., when $A$ is of full rank $k, A$ can be inverted in order to obtain an estimate $\widehat{\mathbf{z}}$ of $\mathbf{z}$

$$
\widehat{\mathbf{z}}=A^{-1} \mathbf{p} .
$$

and $\widehat{x}$ is then

$$
\widehat{\mathbf{x}}=\alpha \widehat{\mathbf{z}}+\beta .
$$

\section{B. Network-coded packets are missing}

When not enough network-coded packets are received, i.e., when $\operatorname{rank}(A)<k$, the inversion (5) is not possible. Let $\mathbf{z}_{0}=$ $\left(z_{1}, \ldots, z_{m}\right)^{T}$ and $\mathbf{z}_{1}=\left(z_{m+1}, \ldots, z_{k}\right)^{T}$ be a partition of the entries of $\mathbf{z}$ such that the corresponding partition of the coding matrix

$$
A=\left[A_{0} A_{1}\right]
$$

leads to

$$
\mathbf{p}=A_{0} \mathbf{z}_{0}+A_{1} \mathbf{z}_{1},
$$

with $A_{0}$ of full rank $m$. Such partition always exist up to a suitable permutation of the columns of $A$ and the unknown entries of $\mathbf{z}$, since the rank of $A$ is $m$.

Our aim is now to provide a MAP estimate of $\mathbf{z}_{1}$ using $\mathbf{p}$ and $\Sigma$

$$
\begin{aligned}
\widehat{\mathbf{z}}_{1} & =\arg \max _{\mathbf{z}_{1}} P\left(z_{m+1}, \ldots, z_{k} \mid \mathbf{p}\right) \\
& =\arg \max _{\mathbf{z}_{1}} \sum_{\mathbf{z}_{0}} P\left(z_{1}, \ldots, z_{k} \mid \mathbf{p}\right) \\
& =\arg \max _{\mathbf{z}_{1}} \sum_{\mathbf{z}_{0}} P\left(\mathbf{p} \mid z_{1}, \ldots, z_{k}\right) P\left(z_{1}, \ldots, z_{k}\right) .
\end{aligned}
$$

Assuming that the quantization step $\Delta$ is small, one can write

$$
P\left(z_{1}, \ldots, z_{k}\right)=f\left(Q^{-1}\left(z_{1}\right), \ldots, Q^{-1}\left(z_{k}\right)\right) \Delta^{k}
$$

where $f$ is the a priori pdf of $\mathbf{x}$

$$
f(\mathbf{x})=\frac{1}{(2 \pi)^{k / 2}|\Sigma|^{1 / 2}} \exp \left(-\frac{1}{2} \mathbf{x}^{T} \Sigma^{-1} \mathbf{x}\right) .
$$


Using (8) and the fact that $A_{0}$ is of full rank $m$, one gets

$$
\mathbf{z}_{0}=A_{0}^{-1}\left(\mathbf{p}-A_{1} \mathbf{z}_{1}\right) .
$$

Combining (12) and (14) in (11), and since $\Delta^{k}$ is constant, one gets

$$
\begin{aligned}
\widehat{\mathbf{z}}_{1}= & \arg \max _{\mathbf{z}_{1}} P\left(\mathbf{p} \mid A_{0}^{-1}\left(\mathbf{p}-A_{1} \mathbf{z}_{1}\right), \mathbf{z}_{1}\right) \\
& f\left(Q^{-1}\left(z_{1}\right), \ldots, Q^{-1}\left(z_{k}\right)\right) .
\end{aligned}
$$

For the first term in (15), one has

$P\left(\mathbf{p} \mid A_{0}^{-1}\left(\mathbf{p}-A_{1} \mathbf{z}_{1}\right), \mathbf{z}_{1}\right)=\left\{\begin{array}{ll}1 & \text { if } \mathbf{p}=A \\ 0 & \text { else. }\end{array}\left(\begin{array}{c}A_{0}^{-1}\left(\mathbf{p}-A_{1} \mathbf{z}_{1}\right) \\ \mathbf{z}_{1}\end{array}\right)\right.$

Consequently, using the fact that $Q^{-1}\left(z_{i}\right)=\alpha z_{i}+\beta$, one can write

$$
\widehat{\mathbf{z}}_{1}=\arg \max _{\mathbf{z}_{1}} f\left(\alpha\left(A_{0}^{-1}\left(\mathbf{p}-A_{1} \mathbf{z}_{1}\right)\right)+\beta, \alpha \mathbf{z}_{1}+\beta\right)
$$

Using (13), (16) may be written as

$$
\widehat{\mathbf{z}}_{1}=\arg \min _{\mathbf{z}_{1}}\left[\begin{array}{c}
\alpha \mathbf{z}_{0}+\beta \\
\alpha \mathbf{z}_{1}+\beta
\end{array}\right]^{T} \Sigma^{-1}\left[\begin{array}{c}
\alpha \mathbf{z}_{0}+\beta \\
\alpha \mathbf{z}_{1}+\beta
\end{array}\right] .
$$

Obtaining $\widehat{\mathbf{z}}_{1}$ requires the solution of a quadratic optimization problem. The main difficulty comes from the fact that the evaluation of $A_{0}^{-1}\left(\mathbf{p}-A_{1} \mathbf{z}_{1}\right)$ involves operations in $\mathbb{F}_{q}$, whereas all other operations have to be done in $\mathbb{R}$. To address this issue, assuming that $q$ is prime, (17) is rewritten by introducing a vector of slack variables $\mathbf{s} \in \mathbb{Z}^{m}$ as follows

$$
\widehat{\mathbf{z}}_{1}=\arg \min _{\mathbf{z}_{1}}\left[\begin{array}{c}
\alpha \mathbf{z}_{0}^{\prime}+\beta \\
\alpha \mathbf{z}_{1}+\beta
\end{array}\right]^{T} \Sigma^{-1}\left[\begin{array}{c}
\alpha \mathbf{z}_{0}^{\prime}+\beta \\
\alpha \mathbf{z}_{1}+\beta
\end{array}\right]
$$

with

$$
\mathbf{z}_{0}^{\prime}=A_{0}^{-1}\left(\mathbf{p}-A_{1} \mathbf{z}_{1}\right)+q \mathbf{s}
$$

and with the constraints

$$
\mathbf{z}_{0}^{\prime} \in\{0, \ldots, q-1\}^{m} .
$$

The inverse $A_{0}^{-1}$ of $A_{0}$ is still computed in $\mathbb{F}_{q}$. Now all operations in (19) may be done in $Z$.

The solution of (18) with the constraints (19) and (20) requires now the solution of an Integer Quadratic Problem (IQP). This kind of minimization problem can be modeled using AMPL [9]. Since the IQP problem involves only convex quadratic forms, CPLEX [1] may be used to solve it.

\section{Simulations Results}

We consider a WSN consisting of $k=10$ sensor nodes. The nodes are uniformly spread over a square of $1 \mathrm{~km}$ width. All sensors located within a circle of radius $d_{0}=1 \mathrm{~km}$ centered in the sensor $s_{i}$ are neighbors of $s_{i}$ and can directly communicate with $s_{i}$. The set of neighbors of $s_{i}$ is $\mathcal{N}\left(s_{i}\right)$. The correlation between measurements is assumed to be represented by (1), with $\sigma^{2}=0.9$ and $\lambda=0.4 \mathrm{~km}^{-2}$.

A very simple transmission protocol is considered. Time is slotted, all sensors are synchronized. In the time slots

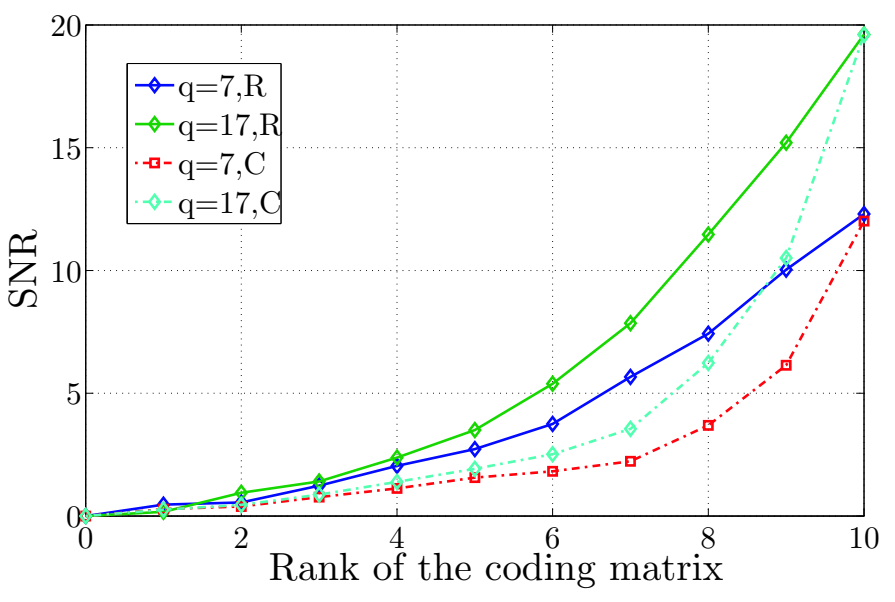

Fig. 2. SNR as a function of the number of linearly independent packets available at the sink for $q=7$ and $q=17$, with the proposed approach (R) and with a conventional Gaussian elimination (C)

devoted to the $i$-th sensor, only $s_{i}$ is allowed to transmit, the other sensors are listening. The $i$-th sensor performs a linear combination over $\mathbb{F}_{q}$ of the packet containing its own quantized measurement and the packets it has already received from its neighbors. The resulting network-coded packet is then transmitted and received by all sensors in $\mathcal{N}\left(s_{i}\right)$. Among packets reaching the sink, only linearly-independent packets are kept.

Simulations are averaged over 1000 realizations of the network and of the data samples.

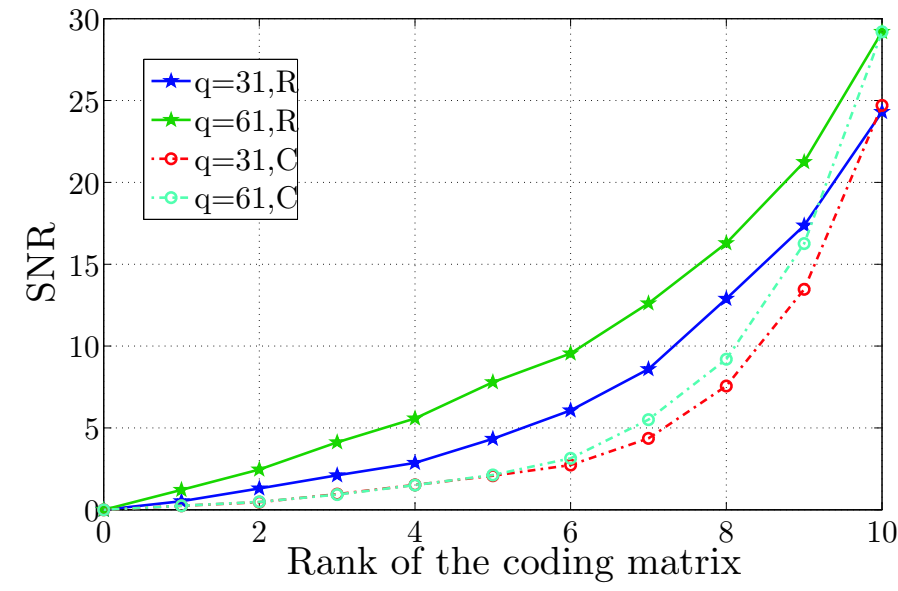

Fig. 3. SNR as a function of the number of linearly independent packets available at the sink for $q=7$ and $q=17$, with the proposed approach (R) and with a conventional Gaussian elimination (C)

Figures 2 and 3 compares the average Signal-to-Noise Ratio (SNR) obtained at the sink as a function of the number of available linearly independent packets, i.e., of the rank of $A$, and for $q \in\{7,17,31,61\}$. The estimation method presented in Section III-B (denoted R) is compared to a classical Gaussian elimination performed on the received packets (denoted C). This Gaussian elimination may work since uncoded packets may reach the data processing sink, measurements not 
estimated in this case are replaced by their mean. The SNR gracefully increases with the number of received packets, $\mathrm{R}$ outperforming $\mathrm{C}$, i.e., for the same SNR, less measurements are required.

The proportion of quantized data samples $z_{i}$ erroneously estimated as a function of the rank of $A$ for $q \in\{7,17,31,61\}$ is represented in Figures 4 and (5). Consistently with the results in Figures 2 and 3, the rank of $A$ required to obtain a given probability of error is smaller with $\mathrm{R}$ than with $\mathrm{C}$. About 2 to 3 less measurements are necessary in average.

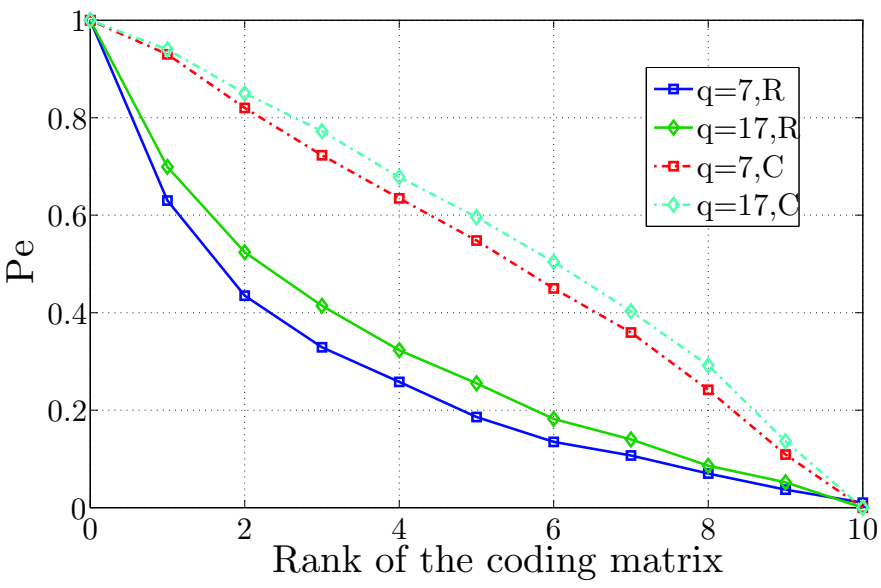

Fig. 4. Proportion of erroneously reconstructed quantized samples as a function of the rank of $A$ sink for $q=7$ and $q=17$, with the proposed approach (R) and with a conventional Gaussian elimination (C)

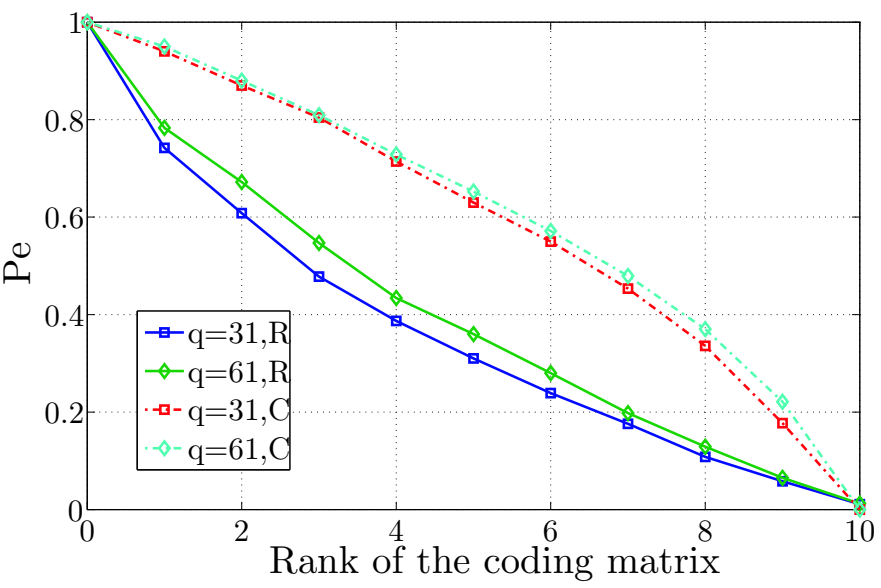

Fig. 5. Proportion of erroneously reconstructed quantized samples as a function of the rank of $A$ sink for $q=31$ and $q=61$, with the proposed approach ( $\mathrm{R}$ ) and with a conventional Gaussian elimination (C)

\section{CONCLUSIONS}

In this paper, we investigate the problem of transmission of spatially correlated sources when RLNC is performed through the transmission network. We provide a MAP estimator in order to reconstruct the transmitted packets using the spatial correlation between quantized measurements stored in the network-coded packets. The reconstruction quality gracefully increases with the number of received packets. For comparable probability of reconstruction error or SNR, less packets are required using the proposed estimator than with a conventional Gaussian-elimination based estimator.

Further experiments with more network nodes and various communication range between sensors will be provided in the final version of the paper.

\section{ACKNOWLEDGMENTS}

The authors would like to thank Leo Liberti for useful discussions. This work is partially supported by DIM-LSC NC2, DIM-LSC SWAN, and by Project 39/2012/H/NDT granted by the Ministry of Science and Technology of Vietnam. Michel Kieffer is partly supported by the Institut Universitaire de France.

\section{REFERENCES}

[1] ILOG CPLEX 11.0 User's Manual.

[2] R. Ahlswede, N. Cai, S.-Y. R. Li, and R. W. Yeung. Network information flow. IEEE Transactions on Information Theory, vol. 46, no. 4, pp. 12041216, July 2000.

[3] J. Barros and S. D. Servetto. Network information flow with correlated sources. in IEEE Trans. on Information Theory, vol. 52, no. 1, pp. 155-170, January 2006.

[4] E. Candes and T. Tao. Near-optimal signal recovery from random projections: Universal encoding strategies? IEEE Transactions Information Theory, vol. 52, no. 12, pp. 5406-5425, December 2006.

[5] S. Chachulskit, M. Jennings, S. Katti, and D. Katabi. Trading structure for randomness in wireless opportunistic routing. In in SIGCOMM, 2007.

[6] P. Chou, Y. Wu, and K. Jain. Practical network coding. In Proc. of the 41-st Allerton Conference, Monticello, IL, 2003.

[7] R. Cristescu, B. Beferull-Lozano, and M. Vetterli. Networked slepianwolf: theory, algorithms, and scaling laws. IEEE Transactions on Information Theory, 51(12):4057 - 4073, dec. 2005.

[8] D. L. Donoho. Compressed sensing. IEEE Transactions Information Theory, vol. 52, no. 4, pp. 1289-1306, 2006.

[9] R. Fourer and D. Gay. The AMPL Book. Duxbury Press, 2002.

[10] T. Ho, M. Medard, M. Effros, and R. Koetter. Network coding for correlated sources. In in IEEE Int. Conf. Inf. Sciences and Syst. (CISS ), Princeton, NJ, USA, March 2004.

[11] L. Howard and P. G. Flikkema. Integrated source-channel decoding for correlated data-gathering sensor networks. In in Proc. IEEE Wireless Communications and Networks Conference (WCNC), Las Vegas, NV, USA, Apr. 2008

[12] S. Katti, D. Katabi, N. Hu, H.S. Rahul, and M. Medard. Xors in the air: practical wireless network coding. IEEE/ACM Transactions on Networking (TON), vol. 16, no. 4, June 2008.

[13] S-Y. R. Li, R. W. Yeung, and N. Cai. Linear network coding. IEEE Transactions on Information Theory, vol. 49, pp. 371-381, 2003.

[14] S. Shintre, S. Katti, and S. Jaggi. Real and complex network codes: Promises and challenges. In Proc. Fourth Workshop on Network Coding, Theory and Applications (NetCod 2008)., pages 1-6, Hong-Kong, China, 2008.

[15] D. Slepian and J.K. Wolf. Noiseless coding of correlated information sources. IEEE Transactions on Information Theory, vol. 19, no. 4, pp. 471-480, 1973.

[16] E. Martinian T. P. Coleman and E. Ordentlich. Joint source-channel coding for transmitting correlated sources over broadcast networks. IEEE Transactions on Information Theory, vol. 55, no. 8, pp. 38643868, August 2009.

[17] Y. Wu, V. Stankovic, Z. Xiong, and S. Y. Kung. On practical design for joint distributed source and network coding. In in First Workshop on Network Coding, Theory, and Applications, NetCod, Riva del Garda, Italy, April 2005

[18] A. Wyner and J. Ziv. The rate-distortion function for source coding with side information at the decoder. IEEE Transactions Information Theory, vol. 22, no. 1, pp. 1-10, 1976. 